
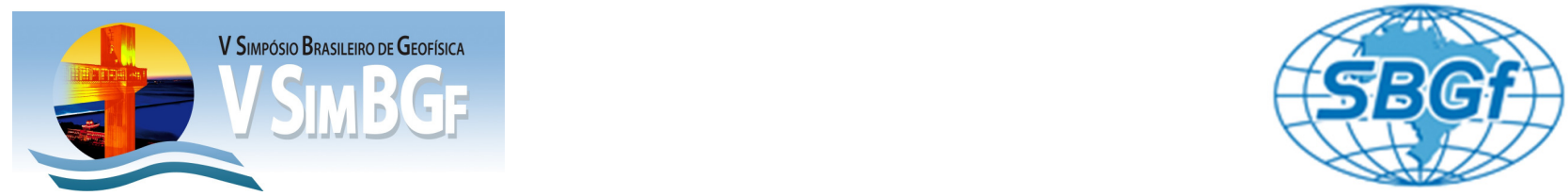

\title{
Gradiente Geotérmico de São Domingos do Prata na Região Centro-Leste do Estado de Minas Gerais.
}

\author{
Virgílio Fagundes M. Sousa (virgilio.fagundes@gmail.com) e Antonio Jorge L. Gomes (antonio.gomes@ufvjm.edu.br) \\ UFVJM - Universidade Federal Dos Vales do Jequitinhonha e Mucuri.
}

Copyright 2012, SBGf - Sociedade Brasileira de Geofísica

Este texto foi preparado para a apresentação no V Simpósio Brasileiro de Geofísica, Salvador, 27 a 29 de novembro de 2012. Seu conteúdo foi revisado pelo Comitê Técnico do V SimBGf, mas não necessariamente representa a opinião da SBGf ou de seus associados. É proibida a reprodução total ou parcial deste material para propósitos comerciais sem prévia autorização da SBGt.

\section{Resumo}

Como parte de um projeto de pesquisa de Energia Geotérmica dos Vales do Jequitinhonha e Mucuri, apresentamos neste trabalho resultados de uma reavaliação do gradiente geotérmico de dois poços da cidade de São Domingos do Prata em Minas Gerais. Estes poços foram perfilados em 1977 e constam da base de dados do Laboratório de Geotermia do Observatório Nacional/MCT do Rio de Janeiro. Para a determinação do gradiente, na nova avaliação, foram utilizados os métodos CVL (convencional) e CBT (temperatura estável de fundo de poço). Foram realizados, ainda, levantamentos de temperaturas mais antigas registradas em superfície, na tentativa de minimizar os efeitos de perturbações térmicas causadas por mudanças climáticas e aquecimento global, no sentido de determinar gradientes térmicos mais consistentes. Os gradientes geotérmicos obtidos estão situados num intervalo de 16,8 e $22,8 \stackrel{\circ}{\circ} \mathrm{C} / \mathrm{km}$. Os dados obtidos indicam que esta região é caracterizada por gradientes geotérmicos relativamente baixos, inclusive 0 local está situado na Província Mantiqueira, região geologicamente estável do tipo pré-cambriana. O valor médio do gradiente geotérmico dos dois locais da cidade de São Domingos do Prata foi de 19,8 $\pm 2,4 \stackrel{\circ}{\circ} / \mathrm{km}$.

\section{Introdução}

Em 2010 foi iniciado em Teófilo Otoni um programa de pesquisa da Energia Geotérmica dos Vales do Jequitinhonha e Mucuri pelo Laboratório de Geotermia da UFVJM - Universidade Federal dos Vales do Jequitinhonha e Mucuri, campus Mucuri Teófilo Otoni, cujo laboratório está em fase de implementação. O projeto tem por objetivo avaliar o potencial geotérmico dos Vales do Jequitinhonha e Mucuri.

A necessidade de reavaliar os dados antigos, para uma nova análise dos gradientes geotérmicos da Província Mantiqueira, surge na tentativa de entendermos melhor o contexto no qual se insere o Complexo Juiz de Fora e consequentemente o seu entorno, no que diz respeito ao gradiente geotérmico.

Neste sentido, vêm sendo compiladas medições de gradientes na região dos Vales do Jequitinhonha e Mucuri e, ainda, a revisão de perfilagens já realizadas a nível regional, visando o melhor entendimento do contexto do Complexo Juiz de Fora.

De acordo com Gomes e Alexandrino (2011) aquela região é caracterizada por baixos valores de gradientes geotérmicos, entretanto, não descartam a possibilidade de anomalias geotérmicas.

Os dados foram complementados utilizando-se como medidas de temperatura da superfície, valores de médias climatológicas antigas (Serra, 1955), na tentativa de minimizar os efeitos de perturbações térmicas causadas por mudanças climáticas e aquecimento global, diminuindo o grau de incerteza na determinação do gradiente geotérmico (Gomes, 2009).

$\mathrm{Na}$ Figura (1) apresentamos a localização regional do Município de São Domingos do Prata em Minas Gerais.

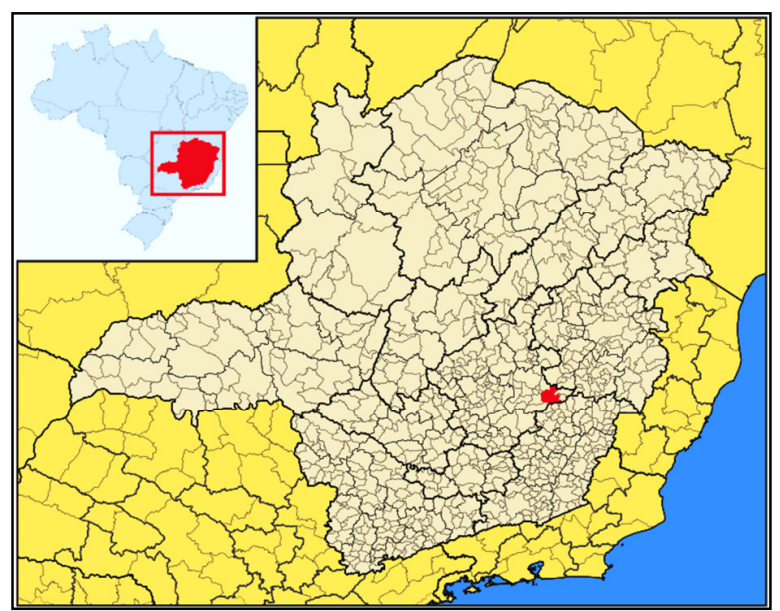

Figura 1 - Mapa regional com a localização do Município de São Domingos do Prata situado na região centro-leste do Estado de Minas Gerais.

\section{Metodologia}

Para a determinação dos gradientes geotérmicos da área de estudo, foram utilizados dois métodos distintos, respectivamente, o método convencional (CVL) e o de temperatura estável do fundo de poço (CBT).

As informações sobre as temperaturas em subsuperfície constituem o acervo básico para avaliação de recursos geotermais. Há dois conjuntos de dados sobre as temperaturas em profundidade: medidas diretas e estimativas indiretas (Bolditzar, 1958; Bullard, 1965; Bodvarsson, 1974; Jessop et al, 1976; Haenel e Mongelli, 1988; Fournier, 1991).

As medidas de temperatura foram efetuadas como uso de sensores tipo termistor. Os termistores são semicondutores que apresentam propriedades físicas 
específicas, onde a resistência diminui com o aumento da temperatura (Gomes, 2004).

O método convencional (CVL) é utilizado para determinação do gradiente geotérmico através de ajuste linear dos dados de perfilagem para um intervalo selecionado. $O$ intervalo deve estar preferencialmente livre de qualquer processo de perturbação que seja capaz de afetar o regime geotérmico local. O uso deste método é adotado para casos onde as camadas geológicas são lateralmente homogêneas, de propriedades térmicas constantes e possuem dimensões físicas bem definidas em relação aos intervalos das medidas.

Desta forma, a profundidade foi considerada como variável independente e a temperatura como variável dependente.

No presente caso, para um conjunto de $\mathrm{N}$ pares de dados (zi, Ti) os coeficientes são o gradiente térmico $(\Gamma)$ e o intercepto $\left(T_{0}\right)$, dada por:

$$
\begin{gathered}
\Gamma=\frac{N \sum z_{i} T_{i}-\sum z_{i} \sum T_{i}}{N \sum z_{i}^{2}-\left(\sum z_{i}\right)^{2}} \\
T_{0}=\frac{\sum z_{i}^{2} \sum T_{i}-\sum z_{i} \sum z_{i} T_{i}}{N \sum z_{i}^{2}-\left(\sum z_{i}\right)^{2}}
\end{gathered}
$$

O método de temperatura estável de fundo do poço (CBT) é utilizado para casos, em que os fluxos de fluidos no interior do poço, perturbam o regime térmico condutivo, impossibilitando desta forma a determinação pelo método convencional do gradiente geotérmico. Nesses casos, uma variante do procedimento conhecido como 'método de fundo do poço' (BHT), proposto por Carvalho e Vacquiers (1977) e Carvalho (1981) e foi utilizado por Hamza e Muñoz (1996), Gomes e Hamza (2004, 2006 e 2009), Hamza et al (2005), Alexandrino e Hamza (2008), Hamza et al (2010).

O gradiente pelo CBT é determinado pela seguinte relação:

$$
T_{C B T}=T_{0}+q \sum_{i=1}^{N} R_{i} h_{i}
$$

onde $R_{i}$ é a resistividade térmica da camada $i$. O termo do somatório se refere à resistência térmica cumulativa das formações presentes até o fundo do poço onde foi efetuada a medida de temperatura.

\section{Geologia e Geofísica Regional}

A região de São Domingos do Prata onde foram realizadas as perfilagens térmicas consiste de unidades geológicas pertencentes à Província Mantiqueira, que é uma entidade geotectônica instalada a leste dos crátons São Francisco e Rio da Prata. Com uma extensão de cerca de $3.000 \mathrm{~km}$ ao longo da costa atlântica, vai de Montevidéu (Uruguai) ao Sul da Bahia (Brasil), guardando registros de uma complexa evolução do neoproterozóico na América do Sul (900-520 Ma), segundo a obra citada (CPRM, 2003).

A Província Mantiqueira é formada por gnaisses, com intrusões de granitóides e rochas básicas; é composta por terrenos granito-gnáissicos constituídos por tonalitos migmatizados e gnaisses granodoiríticos (Noce, 1995). Nesses gnaisses encontram-se intrusões de vários corpos granitoides e rochas básicas e metabásicas provenientes de diferentes fases de acresção mantélica ou retrabalhamento crustal (Carneiro,1992; Noce, 1995).

$\mathrm{Na}$ Figura (2) apresenta-se um recorte, com a localização da cidade de São Domingos do Prata, inserida no contexto geológico da Província Mantiqueira (CPRM, 2003).

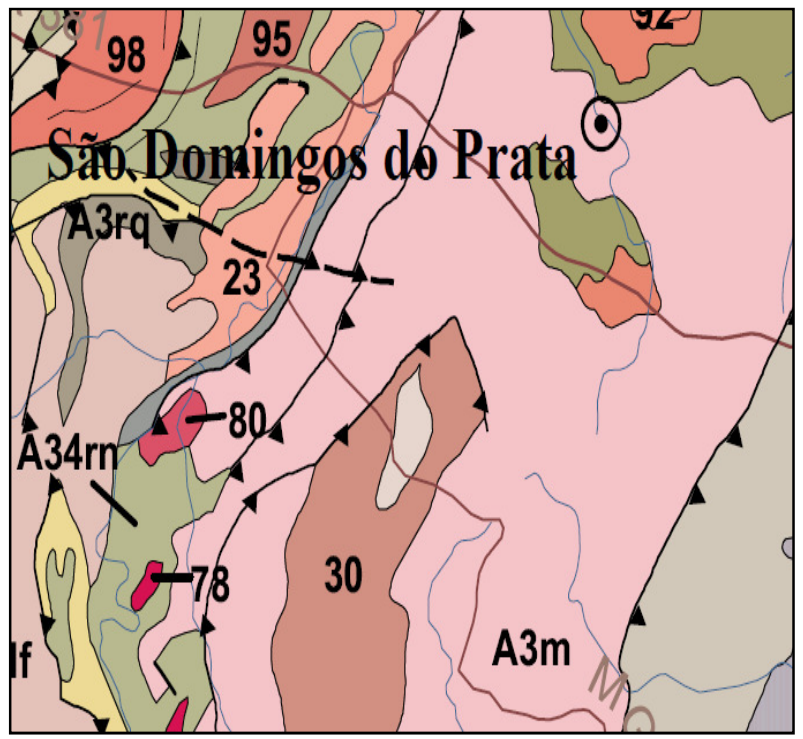

Figura 2 - Geologia de São Domingos do Prata - A3m Província Mantiqueira (recortado de CPRM, 2003).

O clima de São Domingos do Prata, segundo o sistema de classificação de Koppen, é do tipo Aw - tropical úmido, com temperatura média de $21,1^{\circ} \mathrm{C}$, apresentando todos os meses temperaturas médias acima de $18^{\circ} \mathrm{C}$ e estiagem no inverno. Tem-se março como o mês mais quente, apresentando temperaturas de $23,3^{\circ} \mathrm{C}$ e julho como o mais frio, com temperaturas de $18,1^{\circ} \mathrm{C}$ (INMET,2002).

Esta escolha deve-se às baixas perturbações da temperatura de superfície daquela época. Ainda que as medidas não tenham a precisão que as medidas na atualidade, as mesmas, estavam livres do aquecimento industrial do Brasil que teve início após os anos de 1950, e ainda, não muito afetadas pelo aquecimento global iniciado na Revolução Industrial (Gomes, 2009). Desta forma, neste trabalho, utilizamos o valor da temperatura de $17^{\circ} \mathrm{C}$ para $\mathrm{o}$ ajuste da temperatura média de superfície, sendo este valor menor que as médias observadas mais recentemente. Neste trabalho, utilizamos para o método CBT a temperatura média de superfície de $17^{\circ} \mathrm{C}$ com base no Atlas Climatológico do Brasil (Serra, 1955). 


\section{Resultados}

O conjunto de dados utilizado para a reavaliação do gradiente geotérmico em São Domingos do Prata consistiu em dados experimentais, resultante das medições do ano de 1977, integrantes da base de dados do Laboratório de Geotermia do Observatório Nacional/MCT do Rio de Janeiro e de publicações diversas (Hamza e Muñoz, 1996; Hamza et al, 2005; Gomes, 2009, e Gomes e Alexandrino, 2011).

No ano de 1977 um grupo de pesquisa do Observatório Nacional orientado por Hamza fez medições de gradiente na cidade de São Domingos do Prata - Minas Gerais, utilizando o método convencional (CVL) e temperatura estável de fundo do poço (CBT), onde utilizou-se medida da temperatura em superfície para cálculo do gradiente com o mesmo valor deste trabalho. Porém, para que seja levada em conta nos cálculos a influência de tal temperatura na geologia, faz-se o cálculo da média anual da temperatura; na época estimada em $17^{\circ} \mathrm{C}$.

$\mathrm{Na}$ tentativa de reavaliarmos os resultados encontrados, realizamos pesquisas nas bases de dados disponibilizadas na internet pelo INMET no ano de 2012, e com o Atlas climatológico de Serra (1955).

Os resultados obtidos com as novas avaliações encontrados na determinação do gradiente geotérmico de cada poço de São Domingos do Prata, são apresentados no final deste trabalho nas figuras (3) e (4). Nestes gráficos são mostrados os ajustes lineares e também estão especificamente traçados os limites de temperatura com a equação correspondente do gradiente geotérmico e seu intercepto.

Nesse contexto apresentamos na Tabela (1) os resultados de gradiente obtidos em cada poço e por tipo de método utilizado.

Tabela 1 - Resultados do gradiente geotérmico avaliado em São Domingos do Prata por método utilizado.

\begin{tabular}{|c|c|c|c|}
\hline \multirow{2}{*}{ Poço } & \multicolumn{3}{|c|}{ Gradiente Geotérmico (C/Km) } \\
\cline { 2 - 4 } & Tipo & Valor & $\begin{array}{c}\text { Desvio } \\
\text { Padrão }\end{array}$ \\
\hline C-422208-01 & CBT & 22,8 & 3,05 \\
\hline \multirow{2}{*}{ C-422208-02 } & CBT & 18,1 & 0,68 \\
\cline { 2 - 4 } & CVL & 16,8 & 0,17 \\
\hline
\end{tabular}

\section{Conclusões}

Os valores do gradiente geotérmico obtidos pelos métodos CVL e CBT para o Município de São Domingos do Prata, apresentaram valores situados num intervalo entre 16,8 e $22,8 \stackrel{\circ}{\circ} \mathrm{C} / \mathrm{km}$.

O poço C-422208-02 foi o poço de maior profundidade e o que apresentou valores de gradiente CVL de 16,8 $\stackrel{\circ}{ } \mathrm{C} / \mathrm{km} \pm 0,17^{\circ} \mathrm{C} / \mathrm{km}$.

Outra análise do poço C-422208-02 com o método CBT apresentou valor de gradiente de $18,1{ }^{\circ} \mathrm{C} / \mathrm{km} \pm 0,68$ ${ }^{\circ} \mathrm{C} / \mathrm{km}$, muito próximo do valor $\mathrm{CVL}$, dando consistência aos valores encontrados.
Resultados obtidos através do método CBT no poço C422208-01 de menor profundidade, indicaram um gradiente geotérmico de $22,8 \pm 3,05^{\circ} \mathrm{C} / \mathrm{km}$.

A temperatura média anual da região que foi utilizada (Serra, 1955) nos ajustes do método CBT está coerente com os valores do ajuste linear CVL em torno de $17^{\circ} \mathrm{C}$.

$\mathrm{O}$ valor médio do gradiente geotérmico em ambos os poços foi de $19,8 \pm 2,4 \stackrel{\circ}{\circ} \mathrm{C} / \mathrm{km}$.

Os novos resultados obtidos de gradiente geotérmico encontrados confirmam os valores anteriores e esperados para as regiões consideradas geologicamente estáveis e do tipo pré-cambrianas, sobretudo neste local pertencentes à Província Mantiqueira.

\section{Agradecimentos}

Agradecemos à equipe do Laboratório de Geotermia do Observatório Nacional/MCT, em especial ao Doutor Valiya Mannathal Hamza.

\section{Referências}

Serra, A., 1955, Atlas Climatológico do Brasil, v1, CNGSM, Rio de Janeiro.

Gomes, A. J. L. ; HAMZA, Valiya Mannathal, 2004. .Avaliação de Recursos Geotermais do Estado do Rio de Janeiro. Rio de Janeiro, Dissertação de Mestrado, Observatório Nacional.

Gomes, A. J. L. ; Hamza, Valiya Mannathal. 2006. Gradiente e Fluxo Geotérmico no Estado do Paraná. In: II Simpósio de Geofísica da SBGf, 2006. II Simpósio Regional de Geofísica da SBGf, Natal.

Gomes, A. J. L. ; Hamza, Valiya Mannathal, 2009. Avaliação de Recursos Geotermais da Bacia do Paraná, Tese de Doutorado, Observatório Nacional.

Gomes, A.J.L. e Hamza, V.M., 2009. Gradiente e Fluxo Geotérmico da Bacia do Paraná, 11th International Congress of the Brazilian Geophysical Society held in August 24-28, Salvador, Brasil, 2009.

Gomes, A. J. L.; Ramalho, A.M. ; Petzold, A.S. ; Santos, G.M.; Silva, J.M.P.; Campos, P.C.O. 2011. Gradiente Geotérmico do Município de Teófilo Otoni no Estado de Minas Gerais. In: 12th International Congress Of The Brazilian Geophysical Society, 2011, Rio de Janeiro Brasil.

Gomes, A. J. L. ; Alexandrino, C., H. 2011. Mapeamento de Gradiente Geotérmico Do Município De Teófilo Otoni No Estado De Minas Gerais. In: 12th International Congress of The Brazilian Geophysical Society, 2011, Rio de Janeiro.

Hamza, V.M. and Muñoz, M., 1996. Heat Flow map of South America, Geothermics, V.I 25, no 6, pp. 599-646.

Hamza, V.M., Silva Dias, F.J.S., Gomes, A.J.L. and Terceros, Z.G.D., 2005. Numerical and Functional Representations of Regional Heat Flow in South America, Physics of the Earth and Planetary Interiors, Volume 152, 4, p.223-256. 


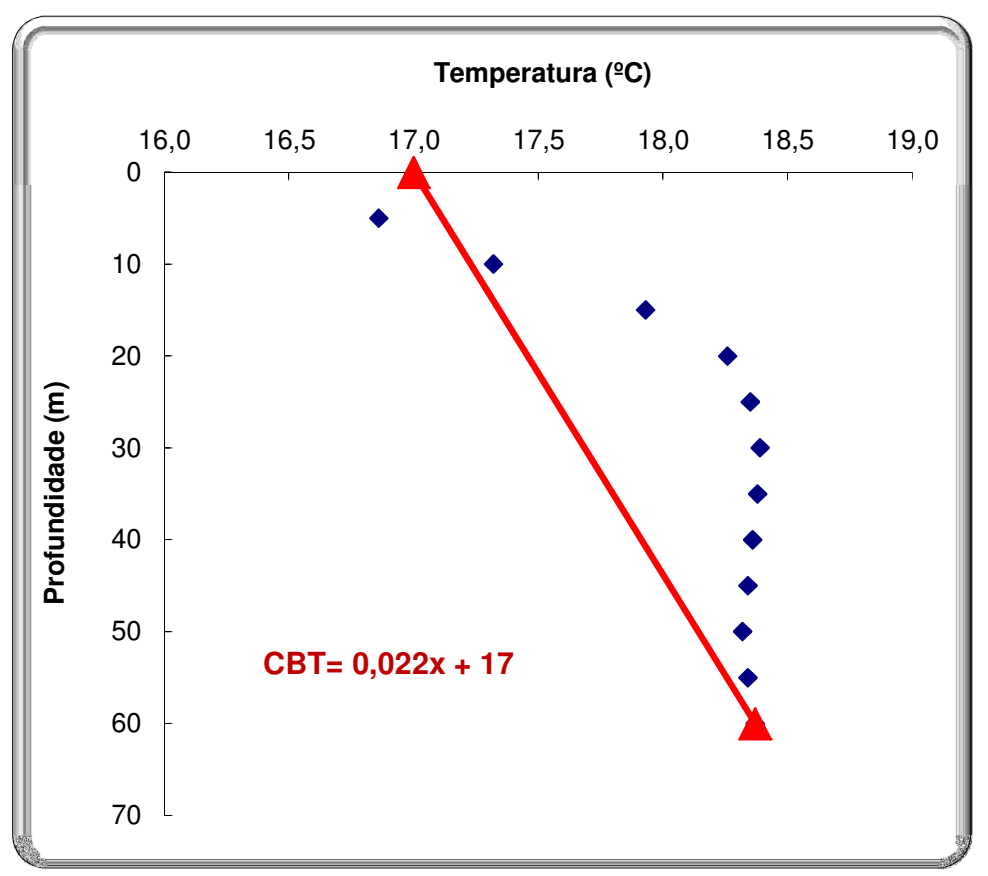

Figura 3 - Perfil térmico do poço C-422208-01 situado em São Domingos do Prata.

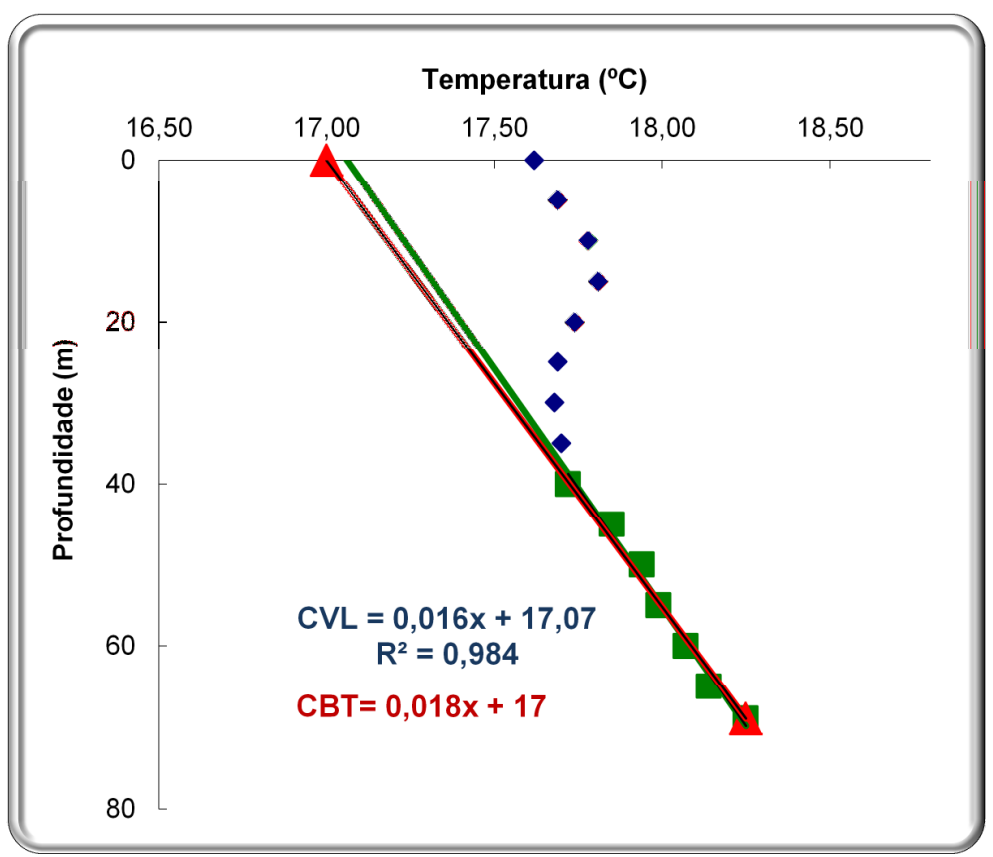

Figura 4 - Perfil térmico do poço C-422208-02 situado em São Domingos do Prata. 